\title{
The psychosocial assessment of deliberate self harm: using clinical audit to improve the quality of the service
}

M Dennis, A Evans, P Wakefield, S Chakrabarti

\begin{abstract}
Objectives-To determine whether simple service initiatives resulted in an improvement in the quality of the psychosocial assessment of adults presenting with deliberate self harm (DSH) by accident and emergency (A\&E) medical staff.

Method-The quality of psychosocial assessment of adults presenting to an $\mathrm{A} \& \mathrm{E}$ department after DSH for a 12 month period was examined using an audit instrument developed from the Royal College of Psychiatrists' standards of service provision for the general hospital management of adult DSH. The results were then compared with a similar audit that had been conducted three years previously. A number of service improvements had been implemented after this first audit.

Results-A total of 1359 episodes of adult DSH were identified. When compared with the previous audit, the frequency of information recorded in the case notes was significantly improved in all areas of the psychosocial assessment $(p<0.001)$ apart from mental state. There were significant changes in treatment between the two audits, with a higher proportion in $1997 / 8(362,26.5 \%)$ assessed by a mental health specialist in the department than in $1994 / 5\left(154,16.5 \% ; \chi^{2}=33, p<0.001\right)$. The frequency of recorded information for those who were not admitted directly to medical or surgical wards was significantly higher for all factors $(p<0.01)$ apart from conscious level and medical history. Conclusions-A substantial improvement in the quality of the psychosocial assessment of adults presenting with DSH by A\&E medical staff was achieved with the introduction of simple service developments. Encouraging staff to use a comprehensive checklist, proved particularly beneficial.

(Emerg Med f 2001;18:448-450)
\end{abstract}

Keywords: deliberate self harm; suicide risk

With significant numbers of patients who self harm being discharged directly home from accident and emergency ( $A \& E$ ) departments it is self evident that a thorough psychosocial assessment should be performed before they leave. ${ }^{1}$ We have previously conducted an audit of the adequacy of the assessment and management of adult deliberate self harm
(DSH) undertaken by A\&E medical staff, ${ }^{2}$ using an audit instrument based upon the Royal College of Psychiatrists' standards of service provision. ${ }^{3}$

Following this audit a number of service improvements were implemented. Firstly, we encouraged staff to use a pre-printed checklist for risk assessment; this was based upon an extensive structured checklist published in the Health Advisory Service review on suicide prevention. ${ }^{4}$ The checklist contains a number of distinct sections: a brief history of the DSH event; previous medical and psychiatric history; social circumstances and background; mental state examination; and a formulation. The mental state examination lists particular points for attention, prompting the clinician to inquire about depressive symptoms, and suicidal thoughts and intentions. Secondly, all new senior house officers were required to attend a one to two hour training seminar conducted by a senior lecturer in psychiatry (MD). The seminar included information on the epidemiology of $\mathrm{DSH}$, the principles and purpose of risk assessment, the psychosocial assessment itself, and the service offered by the local specialist DSH team. This training seminar took place during the new junior medical staffs' routine training programme soon after they started working in the department. Finally, the workings hours of the specialist DSH team were extended from a normal 9 am to $5 \mathrm{pm}$ service to include weekday evenings until $9 \mathrm{pm}$. The DSH team comprises four psychiatric clinical nurse specialists supervised by a consultant liaison psychiatrist. DSH team members will provide mental health assessments on patients presenting with self harm if requested by $A \& E$ medical staff. $A \& E$ medical staff were also encouraged to contact the team, and ask for supervision in assessment of cases if required. Outside the working hours of the specialist DSH team the A\&E medical staff can refer patients for a mental health assessment to an on call senior house officer in psychiatry.

We have now repeated the audit for the 12 month period October 1997 to September 1998.

\section{Method}

All attendances aged 16 and over at the Leicester Royal Infirmary A\&E department with a diagnostic coding of "self inflicted injury" $(n=1598)$, or "psychiatric illness" $(n=330)$ were identified from the computerised database in the department. It is unlikely that episodes of DSH would have been given any 
Table 1 AE E doctor's psychosocial assessment of DSH: a comparison of information recorded in case notes for 1994/95 and 1997/98

\begin{tabular}{|c|c|c|c|c|c|}
\hline \multirow[b]{2}{*}{ Information recorded } & \multicolumn{2}{|c|}{$94 / 95(n=930)$} & \multicolumn{2}{|c|}{$97 / 98(n=1353)$} & \multirow{2}{*}{$\begin{array}{l}p \text { Value } \\
\left(\text { Pearson } \chi^{2}\right)\end{array}$} \\
\hline & Number & $\%$ & Number & $\%$ & \\
\hline Conscious level & 455 & 49 & 954 & 71 & $<0.001$ \\
\hline Psychiatric history & 621 & 67 & 1306 & 97 & $<0.001$ \\
\hline Medical history & 469 & 50 & 1324 & 98 & $<0.001$ \\
\hline Mental state examination & 472 & 51 & 701 & 52 & 0.64 \\
\hline Recent stresses & 515 & 55 & 1020 & 75 & $<0.001$ \\
\hline Previous DSH & 442 & 47 & 982 & 73 & $<0.001$ \\
\hline Employment status & 90 & 10 & 632 & 47 & $<0.001$ \\
\hline Living conditions & 405 & 44 & 790 & 58 & $<0.001$ \\
\hline Threats of DSH & 219 & 23 & 778 & 58 & $<0.001$ \\
\hline Alcohol dependence & 100 & 11 & 656 & 48 & $<0.001$ \\
\hline Illicit drug use & 63 & 7 & 581 & 43 & $<0.001$ \\
\hline Suicide intent & 649 & 70 & 1040 & 77 & $<0.001$ \\
\hline Risk of further DSH & 432 & 46 & 976 & 72 & $<0.001$ \\
\hline
\end{tabular}

other diagnostic coding. The A\&E records were located for 1769 attendances and cases excluded if they were not episodes of DSH as defined by Morgan. ${ }^{5}$ A total of 1359 episodes of adult DSH were identified, 1277 from those coded as "self inflicted injury", and 82 from those coded as "psychiatric illness". The quality of psychosocial assessment was then examined using the same audit instrument as the first audit; this was based upon the Royal College of Psychiatrists statement on the standards of service for the general hospital management of adult $\mathrm{DSH},{ }^{3}$ and has been published previously. ${ }^{2}$ The standards for assessment by non-psychiatric medical staff in the A\&E department requires specific information to be collected and documented in the case notes, in particular concerning; conscious level, psychiatric history and mental state examination, social situation and events, alcohol and drug use, risk, and arrangements for any follow up. An experienced research associate, supervised by the psychiatrists on the audit team, was responsible for examining the case notes and using the audit instrument to assess whether the information required had been recorded. The results of the audit were then compared with those from the first audit (1994/5, $\mathrm{n}=934){ }^{2}$ Case ascertainment in the first audit was similar, although only the notes of those with a discharge diagnosis of "self inflicted injury" had been scrutinised for DSH cases. A 12 month period was chosen for both audits so as to span three rotations of junior medical staff.

Table 2 DSH attendances at AE E: main outcome

\begin{tabular}{|c|c|c|c|c|}
\hline \multirow[b]{2}{*}{ Outcome } & \multicolumn{2}{|l|}{$1994 / 95$} & \multicolumn{2}{|l|}{$1997 / 98$} \\
\hline & $(n=928)$ & $\%$ & $(n=1356)$ & $\%$ \\
\hline Left department before assessed by $\mathrm{A} \& \mathrm{E}$ doctor & 4 & 0.5 & 6 & 0.5 \\
\hline $\begin{array}{l}\text { Discharged home by A\&E doctor with no } \\
\text { psychiatric follow up } \\
\text { Referred for } M H \text { specialist assessment in A\&E }\end{array}$ & 241 & 26 & 361 & 27 \\
\hline $\begin{array}{l}\text { Referred for MH specialist assessment in A\&E } \\
\text { but left before being seen }\end{array}$ & 46 & 5 & 24 & 2 \\
\hline $\begin{array}{l}\text { Discharged by A\&E doctor with psychiatric } \\
\text { outpatient appointment }\end{array}$ & 50 & 5 & 78 & 5.5 \\
\hline $\begin{array}{l}\text { Discharged by MH specialist with psychiatric } \\
\text { outpatient appointment }\end{array}$ & 64 & 7 & 173 & 12.5 \\
\hline Discharged by $\mathrm{MH}$ specialist with no follow up & 45 & 5 & 85 & 6 \\
\hline Admitted to psychiatric ward & 55 & 6 & 146 & 11 \\
\hline Admitted to medical/surgical ward & 423 & 45.5 & 483 & 35.5 \\
\hline
\end{tabular}

Pearson $\chi^{2}=67.1$, d.f. $=7, \mathrm{p}=<0.001 . \mathrm{MH}$ specialist $=$ mental health specialist $(\mathrm{DSH}$ team nurse specialist or on call SHO in psychiatry).
If any patient left the department before, or during the A\&E doctors' assessment they were excluded from the analysis, even though some information may have been recorded from other sources.

\section{Results}

Table 1 shows the comparison of the information recorded in the case notes by $A \& E$ medical staff for the two audits. The assessment checklist was attached to clinical notes in only 549 episodes $(40.4 \%)$ and was fully completed in $73 \%$ of cases. Even when excluding those cases with the checklist present there was still a significant improvement in documentation, in particular for: conscious level $\left(\chi^{2}=\right.$ $72.2, \mathrm{p}<0.001)$; psychiatric history $\left(\chi^{2}=201\right.$, $\mathrm{p}<0.001)$; medical history $\left(\chi^{2}=455, \mathrm{p}<\right.$ $0.001)$; previous $\mathrm{DSH}\left(\chi^{2}=9.6, \mathrm{p}=0.002\right)$; threats of $\mathrm{DSH}\left(\chi^{2}=14, \mathrm{p}<0.001\right)$; chronic alcohol use $\left(\chi^{2}=22, \mathrm{p}<0.001\right)$; illicit drug use $\left(\chi^{2}=7.82, \mathrm{p}=0.005\right)$; suicide intent $\left(\chi^{2}=8.8\right.$, $\mathrm{p}=0.003)$; and risk of further $\mathrm{DSH}\left(\chi^{2}=13.2\right.$, $\mathrm{p}<0.001)$.

There were significant changes in treatment between the two audits, with a higher proportion in 1997/8 (362, 26.5\%) assessed by a mental health specialist (DSH team nurse specialist, or on call SHO in psychiatry) in the department than in $1994 / 5\left(154,16.5 \% ; \chi^{2}=\right.$ $33, \mathrm{p}<0.001)$. In $1997 / 8$ an even smaller proportion of patients were admitted directly to medical and surgical wards than in $1994 / 5$, but interestingly a greater proportion were admitted to psychiatric wards during the period of the second audit (table 2).

When comparing the quality of documentation for episodes admitted to medical or surgical wards with those not admitted, there were significantly higher frequencies of recorded information for those not admitted for all factors $(p<0.01)$ apart from conscious level and medical history. The frequency of information recorded for those not admitted was particularly encouraging-especially for psychiatric history $(97 \%)$, recent stresses $(85 \%)$, previous DSH $(80 \%)$, suicide intent $(87 \%)$, and risk of further DSH (84\%).

For DSH cases attending during the second audit (1997/8), we compared the quality of assessment for cases identified from a coding of "self inflicted injury" with those identified from a coding of "psychiatric illness" and found no significant differences in the documented psychosocial assessment. The improvement in the quality of documentation found in the second audit was therefore not in any way contributed to by the minor differences in case ascertainment.

\section{Discussion}

We have shown a substantial improvement in the quality of documentation of assessment in the second audit compared with the first. By studying the management of three rotations of junior doctor it was unlikely that the improvement was attributable to a cohort effect of one particular group of senior house officers. Considering the service initiatives that occurred it is probable that these interventions were an 
important factor in the improvement that was achieved. Encouraging staff to use a comprehensive checklist, derived from the Health Advisory Service review on suicide prevention ${ }^{4}$ proved particularly beneficial. It was disappointing that the checklist was attached to the notes in only $40 \%$ of cases. However, in some instances the case notes indicated that the sheet had been completed but this was not present in the patients' file. The fact that documentation still improved even when the checklist was absent might indicate that the seminar was having a positive impact and senior house officers had learnt the rudiments of a psychosocial assessment. The fact that the checklist was only fully completed in $73 \%$ of cases was again disappointing, but this may reflect the amount of time required to complete such a comprehensive form.

Crawford and colleagues have also showed an improvement in the quality of DSH assessment by A\&E staff after a one hour teaching seminar. ${ }^{6}$ They encouraged staff to use a proforma, which include the SAD PERSON scale. ${ }^{7}$ The assessment was more likely to be rated as "adequate" if the proforma had been used. The SAD PERSON scale is a much less comprehensive proforma than the checklist used in the Leicester A\&E department. Hockberger and Rothstein ${ }^{8}$ have modified the SAD PERSON scale and shown that by using this non-psychiatric medical staff can confidently identify those patients requiring a specialist assessment. Others have shown the usefulness of risk assessment checklists for house physicians on medical wards. ${ }^{910}$

As previously ${ }^{2}$ there was evidence of selective documentation with much higher rates of recorded information for episodes not admitted directly to medical or surgical wards. It is probable that a proportion of patients admitted directly to general hospital wards would have had a lowering of consciousness hampering their assessment, and A\&E staff would also expect these patients to receive a comprehensive psychosocial assessment during the course of their admission.

There were clear changes in the patterns of management of DSH patients in the second audit compared with the first; the increased rates of assessment by mental health specialists and psychiatric admission during the second audit might indicate better detection of those at risk of suicide. However, in an increasingly litigious climate, it could also be that medical staff are aware of the importance of careful documentation and assessment, and are more likely to refer patients for a specialist assessment if they have any doubts. The reduction in the proportion admitted directly to medical or surgical wards may reflect the increased pressure on general hospital bed usage. Encouragingly, the number of patients who were referred to a mental health specialist but left the department before being seen halved in the second audit (from 46 to 24), and only six left the department before being seen by the $A \& E$ doctor. Crawford and Wessely showed that patients who discharge themselves before the completion of an initial assessment have a considerably increased risk of repeating self harm. ${ }^{11}$

It is not known if improving the assessment of DSH patients in A\&E improves outcome. Further evaluation of whether improved assessment leads to a reduction of repetition of $\mathrm{DSH}$, or subsequent suicide is therefore required. A follow up study of patients receiving an "adequate" psychosocial assessment, and of those referred to mental health services may provide important information.

Contributors

Michael Dennis: proposed the audit project; discussed the core ideas; designed the protocol; developed the audit instrument; supervised the audit assistant and research associate in the data collection; introduced the checklist; gave the teaching seminars; participated in the analysis and interpretation of data; and was responsible for writing the paper. Adrian Evans: discussed the core ideas; participated in the protocol design; helped organise the data collection, introduction of the checklist and teaching seminars; and edited the paper. Penny Wakefield: participated in the data collection, and analysis; and edited the paper. Santanu Chakrabarti: supervised the research associate; participated in the analysis and interpretation of data; and edited the paper. Dr Michael Dennis is guarantor for the paper.

Funding: we thank Leicestershire Health for providing us with financial support.

Conflicts of interest: none.

1 Kapur N, House A, Creed F, et al. Management of deliberate self poisoning in adults in four teaching hospitals: a descriptive survey. BMF 1998;316:831-2.

2 Dennis M, Beach M, Evans PA, et al. An examination of the Dennis $M$, Beach $M$, Evans PA, et al. An examination of the accident and emergency management of

3 The General Hospital Management of Adult deliberate selfharm. A consensus statement on standards for service provision. Council Report CR32. London: The Royal College of Psychiatrists, 1994.

4 Williams R, Morgan HG. Suicide prevention: the challenge confronted. A manual of guidance for the purchasers and providers of mental health care. London: HMSO, 1994.

5 Morgan HG. Death wishes? The understanding and management of deliberate self-harm. Chichester: John Wiley, 1979.

6 Crawford M, Turnball G, Wessely S. Deliberate self harm assessment by accident and emergency staff-an intervention study. F Accid Emerg Med 1998;15:18-22

7 Patterson W, Dohn H, Bird J, et al. Evaluation of suicidal Patterson W, Dohn H, Bird J, et al. Evaluation of suicidal
patients: the SAD PERSONS scale. Psychosomatics 1983; 24:343-5.

8 Hockberger R, Rothstein R. Assessment of suicide potential by nonpsychiatrists using the SAD PERSONS score. 7 Emerg Med 1988;6:99-107

9 Burn W, Edwards J, Machin D. Improving house physicians' assessments of self-poisoning. Br f Psychiatry 1990;157:95100.

10 Blacker C, Jenkins R, Silverstone T. Assessment of deliberate self-harm on medical wards. Psychiatr Bull 1992;16: 262-3.

11 Crawford M, Wessely S. Does initial management affect the rate of repetition of deliberate self-harm? Cohort study. $B M \mathcal{F}$ 1998;317:985. 